\title{
IDENTIFYING PREDICTORS OF A DIFFICULT THYROIDECTOMY
}

\author{
Valerie M. Mok, BS, Sarah C. Oltmann, MD, Herbert Chen, MD, FACS, Rebecca S. Sippel, \\ MD, FACS, and David F. Schneider, MD, MS \\ University of Wisconsin, Department of Surgery, Madison, WI
}

\begin{abstract}
Introduction-A Thyroidectomy Difficulty Scale (TDS) was previously developed that identified more difficult operations, which correlated with longer operative times and higher complication rates. The purpose of this study was to identify pre-operative variables predictive of a more difficult thyroidectomy using the TDS.
\end{abstract}

Methods-A four item, 20-point TDS, was used to score the difficulty of thyroid operations. Patient and disease factors were recorded for each patient. Difficult thyroidectomy (DT) and nondifficult thyroidectomy (NDT) patients were compared. A final multivariate logistic regression model was constructed with significant $(\mathrm{p}<0.05)$ variables from a univariate analysis.

Results-189 patients were scored using TDS. 69 (36.5\%) suffered from hyperthyroidism, 42 (22.2\%) from Hashimoto's, 34 (18.0\%) from thyroid cancer and $36(19.0 \%)$ from multinodular goiter. Among hyperthyroid patients, the DT group had a greater number preoperatively treated with Lugol's potassium iodide ( $81.6 \%$ DT vs. $58.1 \%$ NDT, $\mathrm{p}=0.032$ ), presence of ophthalmopathy (31.6\% DT vs. 9.7\% NDT, $\mathrm{p}=0.028)$ and presence of $(>4 \mathrm{IU} / \mathrm{mL})$ anti-thyroglobulin antibodies (34.2\% DT vs. $12.9 \%$ NDT, p=0.05). Using multivariate analysis, hyperthyroidism (OR 4.35, 95\% CI 1.23-15.36, $\mathrm{p}=0.02$ ), presence of anti-thyroglobulin antibody (OR 3.51, 95\% CI 1.28-9.66, $\mathrm{p}=0.015)$, and high $(>150 \mathrm{ng} / \mathrm{mL})$ thyroglobulin (OR 2.61, 95\% CI 1.06-6.42, $\mathrm{p}=0.037)$ were independently associated with DT.

Conclusion-Using TDS, we demonstrated that a diagnosis of hyperthyroidism, pre-operative elevation of serum thyroglobulin and anti-thyroglobulin antibodies are associated with DT. This tool can assist surgeons in counseling patients regarding personalized operative risk and improve OR scheduling.

Corresponding Author: David F. Schneider, MD, MS, University of Wisconsin, Department of Surgery, 600 Highland Avenue, K4/738 CSC, Madison, WI 53792, schneiderd@ surgery.wisc.edu, 608-263-1387.

Presented at Academic Surgical Congress, San Diego, CA, February 2014.

Author Contributions:

Mok: drafting of manuscript; acquisition of data

Oltmann: critical revision

Chen: critical revision

Sippel: critical revision

Schneider: all elements 


\section{Introduction}

Today, thyroidectomy is a common operation used to treat and/or cure various thyroid disorders. Over ninety thousand thyroid procedures were performed during 2006 in the United States, and the number of thyroid operations continues to rise $(1,2)$. The degree of difficulty as well as length of a thyroid procedure can be influenced by a variety of factors, many of which cannot be assessed until the patient is in the operating room.

In order to have a more objective measurement of difficulty, Schneider and colleagues developed a "Thyroidectomy Difficulty Scale" (TDS) (3). TDS is a four item (vascularity, friability, mobility/fibrosis, gland size), 20-point scale, in which each item is scored on a five-point scale. Immediately following a thyroidectomy, the surgeons completed the TDS. In our previous work, this scale was internally validated, and was shown to have high interrater agreement. Higher TDS scores were noted to correlate with both longer operative times and higher rates of complications (3).

Although the complications from thyroid surgery are rarely fatal, their consequences can be life-long. The main complications associated with thyroidectomy include injury to the recurrent laryngeal nerve, damage to the parathyroid glands, and postoperative hematoma. Recurrent laryngeal nerve injury and hypoparathyroidism have the potential to be life-long, costly issues for a patient (4-6). The incidence of these complications increases with thyroid pathology associated with increased gland size, fibrosis, vascularity, or inflammation (4-6). Although certain disease states such as hyperthyroidism, goiter, and thyroiditis are associated with more difficult thyroidectomies, the degree of difficulty often varies widely, and it is difficult to quantify or predict the level of difficulty of these cases preoperatively ( 3 , 5-11). Surgical risk in thyroidectomy is well studied, but there is no literature regarding the quantification of "difficulty" or an objective measure of "difficulty" for a thyroidectomy (49, 12-15). Difficulty scales have been developed for nephrectomy (16) and choledochotomy (17), but none exist in the area of thyroid surgery. This novel TDS has allowed for quantification of difficulty for thyroidectomy.

If particular patient variables were known to contribute to a more difficult and potentially higher risk thyroid removal, or result in a potentially longer operation, a surgeon would be able to appreciate these factors and take them into consideration when planning a thyroidectomy. This knowledge can improve OR scheduling and preoperative risk counseling. The purpose of this study was to identify objective predictors of a more difficult thyroidectomy.

\section{Methods}

This study involved patients undergoing thyroidectomy by three endocrine surgeons at a high volume tertiary referral center between 2011 and 2013. Patient demographics, preoperative labs, medications, co-morbidities, and postoperative complications were obtained from the prospectively maintained, IRB-approved, Endocrine Surgery database. Surgeons completed the 20-point TDS after the thyroid operation (Figure 1). The difficulty scale includes four factors: vascularity, friability, mobility/fibrosis, and gland size. Each 
factor was graded on a scale from 1-5 with one indicating minimal or normal, and five indicating extensive or significant involvement. An overall score of 20 would indicate the most difficult thyroidectomy (3). Patients undergoing concomitant neck dissection, parathyroidectomy or re-operative thyroidectomies were excluded. Thyroid cancer patients who underwent a central neck lymph node dissection were excluded since their procedures would have a longer operative time. All thyroid cancer patients were grouped together and their histologic classifications were not included in the study. Patients undergoing lobectomy were included in the study, but to account for the difference in time, their operative times were doubled. Operative times were obtained from the electronic operative record and standardized to each surgeon's average time.

In order to categorize each patient as difficult thyroidectomy (DT) or non-difficult thyroidectomy (NDT), a threshold TDS score was determined. The threshold was chosen based on the score that best divided adjusted operating room (OR) times above and below the surgeons' average time. The patient population was divided into subgroups based on their diagnosis of hyperthyroidism, Hashimoto's thyroiditis, thyroid cancer or multinodular goiter. Hyperthyroidism was defined as a patient having TSH $<0.5 \mathrm{mIU} / \mathrm{L}$ (18). A patient was defined as having Hashimoto's thyroiditis if they had an anti-thyroid peroxidase antibody lab value greater than $25 \mathrm{IU} / \mathrm{mL}$. Lab values of anti-thyroglobulin antibody greater than $4 \mathrm{IU} / \mathrm{mL}$ were considered positive for anti-thyroglobulin antibody. A thyroglobulin level greater than $150 \mathrm{ng} / \mathrm{mL}$ was considered "high" thyroglobulin. Hoarseness, defined as voice changes after surgery, and hypoparathyroidism, defined as parathyroid hormone (PTH) below 10, were defined as transient if they resolved within six months, and permanent if the symptoms persisted over six months.

The groups were compared using the student's t-test or Wilcoxon Rank-Sum for continuous variables, chi squared and Fisher's exact test for categorical variables. To identify predictors of DT, a multivariate logistic regression model was constructed with significant $(\mathrm{p}<0.05)$ variables from a univariate analysis. All statistical analyses were performed using STATA v. 12 (StatCorp, College Station, TX). A p value of $<0.05$ was determined to be significant. Data are expressed as mean \pm standard error of the mean, or as number (percentage) as appropriate.

\section{Results}

\section{Patient Demographics}

Over the 24-month study period, a total of 189 consecutive patients were scored using TDS. 146 patients were female $(77.25 \%)$ and the mean age was $49+/-15.0$ years. Within this cohort, 69 patients $(36.5 \%)$ suffered from hyperthyroidism, 42 (22.2\%) from Hashimoto's thyroiditis, 34 (18.0\%) from differentiated thyroid cancer and 36 (19.0\%) from multinodular goiter. Of the 189 patients, $25(13.2 \%)$ were smokers and $3(1.6 \%)$ were pregnant at the time of operation. $72(38.1 \%)$ patients experienced compressive symptoms and $10(5.3 \%)$ had previous radioactive iodine ablation (Table 1). 


\section{Defining Difficult Thyroidectomy}

A score of one in each TDS category (vascularity, friability, mobility/fibrosis, and gland size) would give the minimum total TDS score of 4; a score of five in each category would give the maximum total TDS score of 20. The average total TDS score for all 189 patients was 8.9. Among the 189 patients, the mean score for vascularity was 2.36 , friability was 1.93 , mobility was 2.04 and size was 2.59 .

A threshold TDS score of 10 was chosen to categorize each patient as a difficult thyroidectomy (DT, TDS greater than or equal to 10) or non-difficult thyroidectomy (NDT, less than 10). A score equal to or greater than 10 was chosen because it best divided adjusted OR times above and below the surgeons' average time (Figure 2).

\section{Operative Times}

All operative times were standardized to each surgeon's average time. A relative time of one is equivalent to the surgeon's average time. If an operation took longer than the surgeon's average, the relative time would be greater than one. If an operation took less time than the surgeon's average, the relative time would be less than one. The NDT patients had an average relative time of 0.96 , indicating most NDT patients' operations took less time than the surgeon's average operating time. The DT patients had an average relative time of 1.12, indicating most DT patients' thyroidectomies took longer than the surgeon's average $(\mathrm{p}<0.01)$ as expected based on how these two groups were defined.

\section{Subgroup Analysis}

Of the hyperthyroid patients, the DT group had more than three times as many patients with ophthalmopathy compared to the NDT group ( $\mathrm{p}=0.04$, Table 2$)$. In addition, the hyperthyroid DT group had $23.5 \%$ more patients who were treated with Lugol's potassium iodide drops compared to the hyperthyroid NDT group ( $\mathrm{p}=0.04$, Table 2 ). Lastly, there were almost three times as many patients with anti-thyroglobulin antibodies present in the hyperthyroid DT group than the hyperthyroid NDT group ( $\mathrm{p}=0.05$, Table 2).

Among the Hashimoto's thyroiditis and multinodular goiter subsets, there were no statistically significant differences between the DT and NDT groups.

Of the 34 patients with thyroid cancer, 22 had PTC (64.7\%), 12 (35.3\%) had follicularvariant of PTC, and 1 patient (2.9\%) had FTC. There were no significant differences in TDS scores. In the cancer cohort, those who had a DT had a higher mean preoperative thyroglobulin value compared to NDT patients ( $897 \mathrm{ng} / \mathrm{mL}$ vs. $55 \mathrm{ng} / \mathrm{mL}, \mathrm{p}=0.027$ ). There were no other differences between these two groups in laboratory, demographic, or clinical factors identified in the cancer subset.

\section{Complications}

Of the 189 patients, $40(21.2 \%)$ experienced a postoperative complication. Patients meeting the definition of DT experienced nearly three times the complication rate compared to the NDT group (38.2\% vs. $13.2 \%, \mathrm{p}<0.01$, Table 1$)$. A total of 13 (6.9\%) patients experienced transient hoarseness (voice changes) and of those 13, six (10.0\%) were DT patients 
compared to 7 (5.4\%) in the NDT group ( $\mathrm{p}=0.23$, Table 3). A total of $24(12.7 \%)$ patients experienced transient hypoparathyroidism and of those 24,14 (23.3\%) were DT patients vs. $10(7.8 \%)$ in the NDT group $(\mathrm{p}<0.01$, Table 3$)$. No patients in either group suffered permanent hoarseness or permanent hypoparathyroidism. A total of five (2.6\%) patients experienced a postoperative hematoma and all five $(100 \%)$ were DT patients $(\mathrm{p}<0.01$, Table $3)$.

\section{Multivariate Analysis}

To determine which patient and disease factors were associated with DT, a univariate analysis was first performed using a logistic regression model. Variables found to be significant on univariate analysis $(\mathrm{p}<0.05)$ were then included in the final multivariate model. Variables included in the final model were: age, sex, cancer, hyperthyroidism, Hashimoto's, smoker, TSH less than $0.1 \mathrm{mIU} / \mathrm{L}$, free T4 greater than $1.5 \mathrm{ng} / \mathrm{dL}$, thyroid stimulating immunoglobulin, anti-thyroglobulin antibody, thyroglobulin greater than $150 \mathrm{ng} / \mathrm{mL}$, ophthalmopathy, and greater than or equal to two anti-thyroid medications preoperatively. In this model, three variables were noted to be significant between the DT and NDT groups. Hyperthyroidism (OR 4.35, CI 1.23-15.36, $\mathrm{p}=0.02$ ), the presence of antithyroglobulin antibody greater than $4 \mathrm{IU} / \mathrm{mL}$ (OR 3.51, CI 1.28-9.66, $\mathrm{p}=0.02$ ), and high $(>150 \mathrm{ng} / \mathrm{mL}$ ) thyroglobulin (OR 2.61, CI 1.06-6.42, p=0.04) were independently associated with DT (Table 4).

\section{Discussion}

Objective preoperative measures that predict a more difficult thyroid operation using the TDS were identified. Hyperthyroidism, Graves' ophthalmopathy, pre-operative thyroglobulin, and anti-thyroglobulin antibody were associated with a more difficult thyroidectomy. Knowledge of these predictors of difficulty can aid in operative risk counseling and scheduling operating room time.

TDS is a novel tool; the literature lacks any means for identification of patient or disease factors predictive of a more difficult thyroidectomy. There is, however, research examining factors predictive of complications after surgical procedures in other disciplines $(16,17)$, such as intubation, laparoscopy, cataract surgery, and aneurysm repair (19-23).

Historically, thyroidectomy was performed as an inpatient procedure due to concern for postoperative hematoma or symptomatic hypocalcemia. More recently, the safety and utility of outpatient thyroidectomy has been reported (1,24-26). Outpatient thyroidectomy is considered safe for most patients due to a number of advancements, including PTH testing, calcium supplementation, vocal fold evaluation and the use of intraoperative hemostatic agents and energy devices to reduce bleeding $(24,25)$. In keeping with the efficiency of outpatient operating centers, proper OR scheduling is essential (3). More predictable OR schedules can allow more efficient staffing and cost reduction. Knowing preoperative factors that predict a more difficult operation can assist surgeons with properly scheduling an OR time slot or assigning a case to the inpatient OR due to predicted increased risk and/or operative time $(3,24)$. 
Many of the traditional markers of morbidity and mortality do not necessarily apply to thyroidectomy where the mortality rate approaches zero, but the morbidity can be significant (27-30). The American College of Surgeons (ACS) developed a surgical risk calculator that can be used to estimate the risks of most operations and will allow clinicians and patients to make decisions using empirically derived, patient-specific postoperative risks (31). ACS recognizes the need to be able to estimate customized, patient-specific risks for surgical operations (31). However, none of the items account for the risks specific to thyroidectomy, such as hoarseness or hypocalcemia. With the evolution of pay for performance, it is essential to assess and adjust risk for specific procedures like thyroidectomy, not just overall surgical complications. The TDS helps to identify these higher risk patients specific to thyroidectomy.

Specific to thyroid surgery, the objective data gained from this study, using TDS, can be used to guide preoperative risk counseling so the patient can better know their chance of endocrine-specific complications. For example, hyperthyroid patients with Graves' ophthalmopathy, positive anti-thyroglobulin antibody or those treated with Lugol's SSKI who are undergoing thyroidectomy can be informed that they have a three times higher risk of experiencing complications compared to hyperthyroid patients without these factors.

This study discovered that hyperthyroid patients, most suffering from Graves' disease, had more difficult thyroidectomies. Within the study cohort, a diagnosis of hyperthyroidism was independently associated with a more difficult thyroid operation and more complications. Although thyroidectomy is curative for Graves' disease and morbidity is low at high volume centers (32), Hallgrimsson et al. and Welch et al. both found Graves' disease to be associated with increased morbidity, specifically hypocalcemia, following total thyroidectomy $(33,34)$. Campbell et al. investigated risk factors for hematoma after thyroidectomy and found Graves' disease to be independently associated with hematoma post-thyroidectomy (35). Within this group of hyperthyroid patients, those treated with Lugol's potassium iodide drops, had ophthalmopathy, or had the presence of antithyroglobulin antibodies preoperatively, were noted to have more difficult operations based on the TDS system.

Potassium iodide (KI) is currently administered preoperatively to Graves' disease patients to reduce gland vascularity and diminish blood loss during thyroidectomy, per current American Thyroid Association guidelines (36). Among our study's patient population, Lugol's potassium iodide was selectively administered preoperatively to patients with Graves' disease and those blocked with anti-thyroid medications. It was not given to patients with toxic multinodular goiter or those not requiring anti-thyroid medications. Shinall et al. concluded that when KI is not used there is no appreciable detriment to Graves' disease patient outcomes (36). Even though the use of KI was associated with DT among the hyperthyroid patients, surgeons typically choose to preoperatively administer KI to patients with severe Graves' disease. Therefore, the use of KI may be a marker of patients who had more severe hyperthyroidism preoperatively. However, since KI remained an independent predictor of difficulty, our group is now specifically evaluating the use of KI in hyperthyroidism. Wong et al. found that Graves' ophthalmopathy is associated with 
hypoparathyroidism post-total thyroidectomy, consistent with the finding that hyperthyroid patients with ophthalmopathy experience more complications (37).

In addition, the preoperative anti-thyroglobulin antibody levels can be used to predict a potentially more difficult operation in all patients, and this is in line with studies by McManus and colleagues $(4,14)$. Although much has been published on treatment and operative risk for Graves' disease, Hashimoto's and thyroid cancer patients, there is no published literature that investigates specific preoperative factors that are predictive of a more difficult thyroid operation $(4-11,13-15,18)$. The data found in our study can be used to differentiate which hyperthyroid patients, like those with ophthalmopathy, or which cancer or Hashimoto's patients, will experience a more difficult operation.

Upile et al. discussed "the difficult thyroid" and indicated how obtaining consent for complications that haven't happened yet poses a problem for surgeons (9). Our study describes preoperative factors that predict a more difficult thyroid operation, and since our definition of a difficult thyroid operation (TDS score $>10$ ) was associated with more complications, these factors may predict postoperative complications. Proper informed consent is ethically and legally required for all planned therapies (38); informed consent for a surgery must include the risks, expected benefits and alternatives to treatment, and must also include discussion of the expected postoperative course, including other interventions that may be needed if complications occur $(39,40)$. Since hyperthyroidism, positive antithyroglobulin antibodies and high thyroglobulin are associated with DT, this information should be included during a patient's preoperative counseling, thereby enabling the discussion to be tailored to the individual patient.

There are some limitations to this work. This was a retrospective study from a single high volume center. Therefore, the concept of difficulty may not be generalizable to lower volume centers. Another limitation of this study is the fact that many of the lab values investigated as predicting factors are not routinely drawn. At our center these labs, including anti-thyroglobulin antibodies, anti-thyroid peroxidase antibodies, are drawn under existing, IRB-approved research protocols. Although we do not advocate routine ordering of these labs for all patients undergoing thyroidectomy, this data remains useful since they identify difficult disease processes (thyroiditis, hyperthyroidism), provide threshold values (positive anti-thyroglobulin antibodies, thyroglobulin $>150 \mathrm{ng} / \mathrm{mL}$ ), and can identify potentially difficult cases that might benefit from referral to a high volume center. The next step of this study is to create a simplified prediction model, exploring only standard data that is routinely available.

Blood loss was not included in our difficulty scale because it is a very subjective item. Although these results only represent three surgeons, OR times were normalized to each surgeon's average time to control for differences in time due to a surgeon's experience or technique. Nonetheless, this study was conducted at a high volume, tertiary referral center, and these measures of difficulty may not apply to all surgeons, especially those with lower thyroidectomy volumes. 


\section{Conclusion}

The Thyroid Difficulty Scale was used to identify predictors of difficult thyroidectomy. Patients with hyperthyroidism, thyroglobulin greater than $150 \mathrm{ng} / \mathrm{mL}$, positive antithyroglobulin antibodies, may have a DT associated with longer operative times and increased complications. This information can improve preoperative risk counseling and lead to more efficient scheduling of the operating room.

\section{Acknowledgments}

University of Wisconsin Shapiro Summer Research Program for funding this research project

\section{References}

1. Sun GH, DeMonner S, Davis MM. Epidemiological and economic trends in inpatient and outpatient thyroidectomy in the United States, 1996-2006. Thyroid. 2013; 23(6):727-733. PubMed PMID: 23173840. [PubMed: 23173840]

2. Sosa JA, Hanna JW, Robinson KA, Lanman RB. Increases in thyroid nodule fine-needle aspirations, operations, and diagnoses of thyroid cancer in the United States. Surgery. 2013; 154(6):1420-1427. PubMed PMID: 24094448. [PubMed: 24094448]

3. Schneider DF, Mazeh H, Oltmann SC, Chen H, Sippel RS. A Novel Thyroidectomy Difficulty Scale Correlates With Operative Times. Accepted for publication. World J Surg. 2013 Dec.

4. McManus C, Luo J, Sippel R, Chen H. Is thyroidectomy in patients with Hashimoto thyroiditis more risky? Journal of Surgical Research. 2012; 178(2):529-532. [PubMed: 23043868]

5. Pradeep PV, Ragavan M, Ramakrishna BA, Jayasree B, Skandha SH. Surgery in Hashimoto's thyroiditis: indications, complications, and associated cancers. J Postgrad Med. 2011; 57(2):120 122. [PubMed: 21654133]

6. Shih ML, Lee JA, Hsieh CB, Yu JC, Liu HD, Kebebew E, Clark OH, Duh QY. Thyroidectomy for Hashimoto's thyroiditis: complications and associated cancers. Thyroid. 2008; 18(7):729-734. [PubMed: 18631001]

7. Seifman MA, Grodski SF, Bailey M, Yeung MJ, Serpell JW. Surgery in the setting of Hashimoto's thyroiditis. ANZ J Surg. 2011; 81(7-8):519-523. [PubMed: 22295372]

8. Sundaresh V, Brito JP, Wang Z, Prokop LJ, Stan MN, Murad MH, Bahn RS. Comparative Effectiveness of Therapies for Graves' Hyperthyroidism: A Systematic Review and Network Metaanalysis. J Clin Endocrinol Metab. 2013

9. Upile T, Jerjes W, Mahil J, Tailor H, Balakumar R, Rao A, Qureshi Y, Bowman I, Mukhopadhyay S. How to do it: the difficult thyroid. Head \& Neck Oncology. 2011; 3

10. Bhargav PR, Shekhar S. Surgical Indications for Goiter with Background Hashimoto's Thyroiditis: Institutional Experience. Indian J Surg. 2011; 73(6):414-418. [PubMed: 23204697]

11. Mishra A, Sabaretnam M, Chand G, Agarwal G, Agarwal A, Verma AK, Mishra SK. Quality of Life (QoL) in Patients With Benign Thyroid Goiters (Pre- and Post-Thyroidectomy): A Prospective Study. World J Surg. 2013

12. Bajwa SJ, Sehgal V. Anesthesia and thyroid surgery: The never ending challenges. Indian J Endocrinol Metab. 2013; 17(2):228-234. [PubMed: 23776893]

13. Hegedus L. Treatment of Graves' hyperthyroidism: evidence-based and emerging modalities. Endocrinol Metab Clin North Am. 2009; 38(2):355-371. ix. [PubMed: 19328416]

14. McManus C, Luo J, Sippel R, Chen H. ASSOCIATION FOR ACADEMIC SURGERY Should Patients with Symptomatic Hashimoto's Thyroiditis Pursue Surgery? Journal of Surgical Research. 2011; 170(1):52-55. [PubMed: 21435660]

15. More Y, Shnayder Y, Girod DA, Sykes KJ, Carlisle MP, Chalmers B, Kraemer C, Tsue TT. Factors Influencing Morbidity After Surgical Management of Malignant Thyroid Disease. Annals of Otology Rhinology and Laryngology. 2013; 122(6):398-403. 
16. Mathieu R, Verhoest G, Droupy S, de la Taille A, Bruyere F, Doumerc N, Rischmann P, Vaessen C, Roupret M, Bensalah K. Predictive factors of complications after robot-assisted laparoscopic partial nephrectomy: a retrospective multicentre study. BJU Int. 2013; 112(4):E283-E289. [PubMed: 23879913]

17. Ye X, Hong X, Ni K, Teng X, Xie K. Preoperative factors predicting poor outcomes following laparoscopic choledochotomy: a multivariate analysis study. Can J Surg. 2013; 56(4):227-232. [PubMed: 23883491]

18. Catania A, Guaitoli E, Carbotta G, Bianchini M, Di Matteo FM, Carbotta S, Nardi M, Fabiani E, Grani G, D'Andrea V, Fumarola A. Total thyroidectomy for Graves' disease treatment. Clin Ter. 2013; 164(3):193-196. [PubMed: 23868618]

19. Adnet F, Borron SW, Racine SX, Clemessy JL, Fournier JL, Plaisance P, Lapandry C. The intubation difficulty scale (IDS): proposal and evaluation of a new score characterizing the complexity of endotracheal intubation. Anesthesiology. 1997; 87(6):1290-1297. [PubMed: 9416711]

20. Jamali FR, Soweid AM, Dimassi H, Bailey C, Leroy J, Marescaux J. Evaluating the degree of difficulty of laparoscopic colorectal surgery. Arch Surg. 2008; 143(8):762-767. discussion 8 PubMed PMID: 18711036. [PubMed: 18711036]

21. Dooley IJ, O'Brien PD. Subjective difficulty of each stage of phacoemulsification cataract surgery performed by basic surgical trainees. J Cataract Refract Surg. 2006; 32(4):604-608. PubMed PMID: 16698480. [PubMed: 16698480]

22. Ahanchi SS, Carroll M, Almaroof B, Panneton JM. Anatomic severity grading score predicts technical difficulty, early outcomes, and hospital resource utilization of endovascular aortic aneurysm repair. J Vasc Surg. 2011; 54(5):1266-1272. PubMed PMID: 21803521. [PubMed: 21803521]

23. Seo SH, Lee JG, Yu SB, Kim DS, Ryu SJ, Kim KH. Predictors of difficult intubation defined by the intubation difficulty scale (IDS): predictive value of 7 airway assessment factors. Korean J Anesthesiol. 2012; 63(6):491-497. [PubMed: 23277808]

24. Clark N, Schneider DF, Vrabec S, Bauer PS, Chen H, Sippel RS. Increased efficiency of endocrine procedures performed in an ambulatory operating room. J Surg Res. 2013; 184(1):200-203. PubMed PMID: 23702288; PubMed Central PMCID: PMCPMC3759536. [PubMed: 23702288]

25. Terris DJ, Snyder S, Carneiro-Pla D, Inabnet WB, Kandil E, Orloff L, Shindo M, Tufano RP, Tuttle RM, Urken M, Yeh MW. Force ATASACWT. American thyroid association statement on outpatient thyroidectomy. Thyroid. 2013; 23(10):1193-1202. PubMed PMID: 23742254. [PubMed: 23742254]

26. Snyder SK, Hamid KS, Roberson CR, Rai SS, Bossen AC, Luh JH, Scherer EP, Song J. Outpatient thyroidectomy is safe and reasonable: experience with more than 1,000 planned outpatient procedures. J Am Coll Surg. 2010; 210(5):575-582. 82-84. PubMed PMID: 20421007. [PubMed: 20421007]

27. Revenig LM, Canter DJ, Taylor MD, Tai C, Sweeney JF, Sarmiento JM, Kooby DA, Maithel SK, Master VA, Ogan K. Too frail for surgery? Initial results of a large multidisciplinary prospective study examining preoperative variables predictive of poor surgical outcomes. J Am Coll Surg. 2013; 217(4):665.e1-670.e1. PubMed PMID: 24054409. [PubMed: 24054409]

28. Rodríguez Faba O, Palou J, Breda A, Maroto P, Fernández Gómez JM, Wong A, Villavicencio H. Predictive Factors for Impaired Renal Function following Nephroureterectomy in Upper Urinary Tract Urothelial Cell Carcinoma. Urol Int. 2013 PubMed PMID: 24280761.

29. Bhattacharyya N, Fried MP. Assessment of the morbidity and complications of total thyroidectomy. Arch Otolaryngol Head Neck Surg. 2002; 128(4):389-392. PubMed PMID: 11926912. [PubMed: 11926912]

30. Carter Y, Chen H, Sippel RS. An intact parathyroid hormone-based protocol for the prevention and treatment of symptomatic hypocalcemia after thyroidectomy. J Surg Res. 2013 PubMed PMID: 24144426.

31. Bilimoria KY, Liu Y, Paruch JL, Zhou L, Kmiecik TE, Ko CY, Cohen ME. Development and evaluation of the universal ACS NSQIP surgical risk calculator: a decision aid and informed consent tool for patients and surgeons. J Am Coll Surg. 2013; 217(5):833, 42.e1-43.e1. PubMed PMID: 2405-5383; PubMed Central PMCID: PMCPMC3805776. [PubMed: 24055383] 
32. Liu J, Bargren A, Schaefer S, Chen H, Sippel RS. Total thyroidectomy: a safe and effective treatment for Graves' disease. J Surg Res. 2011; 168(1):1-4. PubMed PMID: 213495453; PubMed Central PMCID: PMCPMC3085652. [PubMed: 21345453]

33. Hallgrimsson P, Nordenström E, Bergenfelz A, Almquist M. Hypocalcaemia after total thyroidectomy for Graves' disease and for benign atoxic multinodular goitre. Langenbecks Arch Surg. 2012; 397(7):1133-1137. PubMed PMID: 22976368. [PubMed: 22976368]

34. Welch KC, McHenry CR. Total thyroidectomy: is morbidity higher for Graves' disease than nontoxic goiter? J Surg Res. 2011; 170(1):96-99. PubMed PMID: 21550063. [PubMed: 21550063]

35. Campbell MJ, McCoy KL, Shen WT, Carty SE, Lubitz CC, Moalem J, Nehs M, Holm T, Greenblatt DY, Press D, Feng X, Siperstein AE, Mitmaker E, Benay C, Tabah R, Oltmann SC, Chen H, Sippel RS, Brekke A, Vriens MR, Lodewijk L, Stephen AE, Nagar S, Angelos P, Ghanem M, Prescott JD, Zeiger MA, Aragon Han P, Sturgeon C, Elaraj DM, Nixon IJ, Patel SG, Bayles SW, Heneghan R, Ochieng P, Guerrero MA, Ruan DT. A multi-institutional international study of risk factors for hematoma after thyroidectomy. Surgery. 2013; 154(6):1283-1291. PubMed PMID: 24206619. [PubMed: 24206619]

36. Shinall MC, Broome JT, Baker A, Solorzano CC. Is potassium iodide solution necessary before total thyroidectomy for graves disease? Ann Surg Oncol. 2013; 20(9):2964-2967. PubMed PMID: 23846785. [PubMed: 23846785]

37. Wong KP, Lang BH. Graves' ophthalmopathy as an indication increased the risk of hypoparathyroidism after bilateral thyroidectomy. World J Surg. 2011; 35(10):2212-2218. PubMed PMID: 21858556, PubMed Central PMCID: PMCPMC3170470. [PubMed: 21858556]

38. Rosenthal MS, Angelos P, Cooper DS, Fassler C, Finder SG, Hays MT, Tendler B, Braunstein GD. Committee ATAEA. Clinical and professional ethics guidelines for the practice of thyroidology. Thyroid. 2013; 23(10):1203-1210. PubMed PMID: 23750916. [PubMed: 23750916]

39. Kinnersley P, Phillips K, Savage K, Kelly MJ, Farrell E, Morgan B, Whistance R, Lewis V, Mann MK, Stephens BL, Blazeby J, Elwyn G, Edwards AG. Interventions to promote informed consent for patients undergoing surgical and other invasive healthcare procedures. Cochrane Database Syst Rev. 2013; 7:CD009445. PubMed PMID: 23832767. [PubMed: 23832767]

40. Wall A, Angelos P, Brown D, Kodner IJ, Keune JD. Ethics in surgery. Curr Probl Surg. 2013; 50(3):99-134. PubMed PMID: 23445722. [PubMed: 23445722] 


\section{Thyroidectomy Difficulty Scale (TDS)}

Patient Name/MRN

Surgeon

Date of Surgery

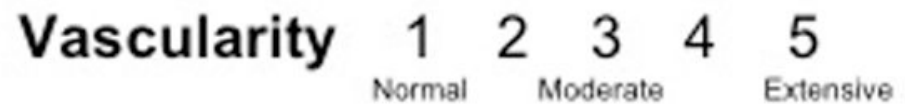

Friability

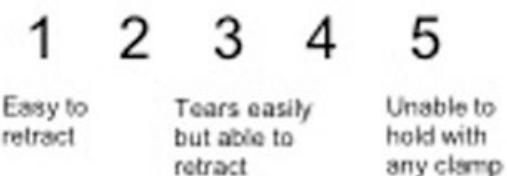

Mobility/

$\begin{array}{lllll}1 & 2 & 3 & 4 & 5\end{array}$

Fibrosis

Eevates Able to retract Fixed in

essily medislywith postioniunable

$\begin{array}{llllll}\text { Gland Size } & 1 & 2 & 3 & 4 & 5\end{array}$

$\begin{array}{lll}\text { Nomal } & \text { Above } & \text { Largeigozer } \\ \text { size } & \text { average } & \end{array}$

Point Total

Comments:

\section{Figure 1. TDS Score Sheet}

The surgeon completed this form after a thyroidectomy. The TDS includes four items: vascularity, friability, mobility/fibrosis, and gland size. Each item is scored on a five-point scale. 


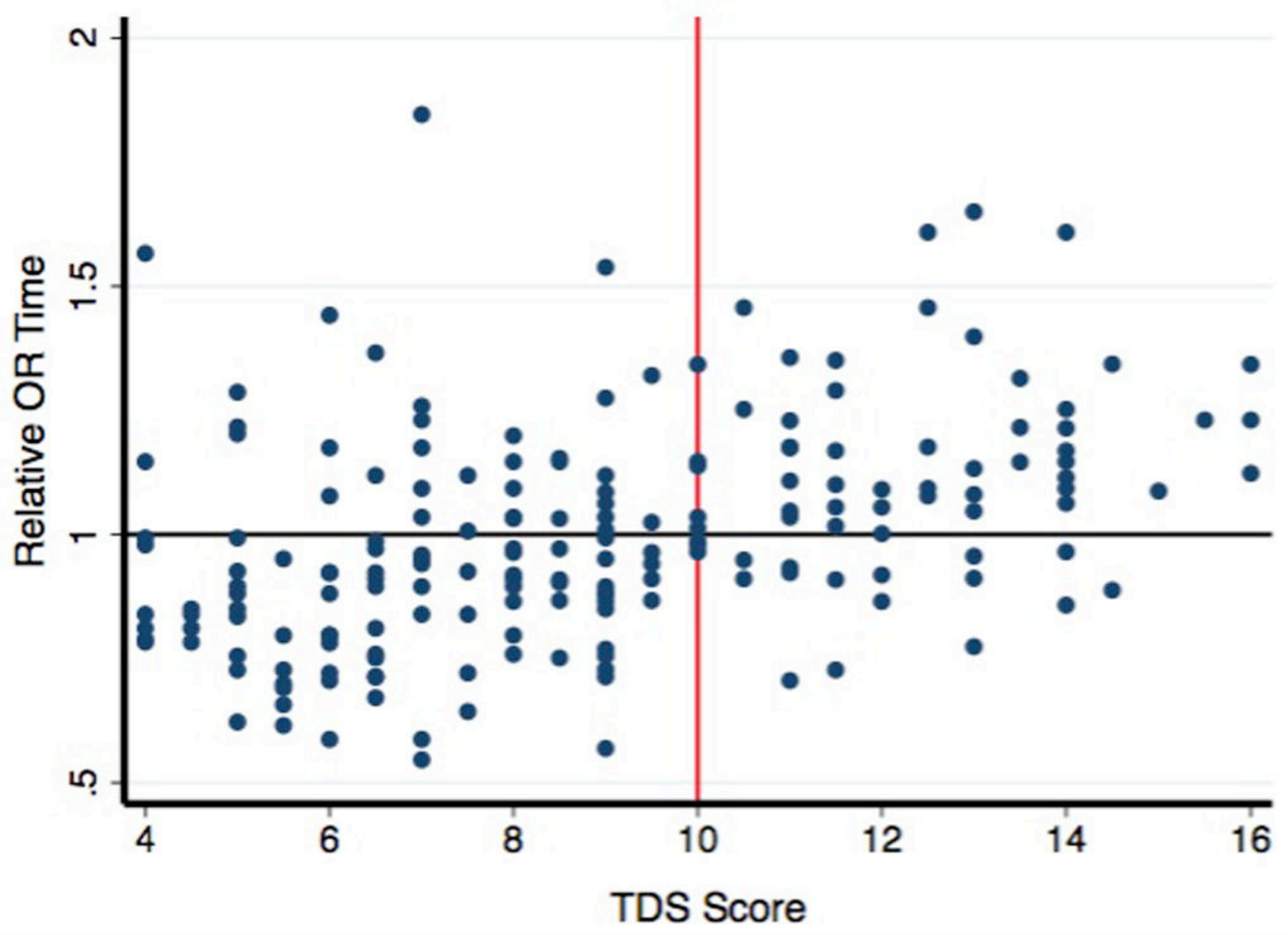

Figure 2. TDS Score vs. Relative OR Time

A relative time of one signifies the surgeon's average time. A TDS threshold of 10 was defined as a difficult thyroidectomy since it best divided operative times above and below each surgeon's average time. 


\section{Table 1}

Patient Demographics $(\mathrm{n}=189)$

\begin{tabular}{lccc}
\hline Variable & NDT & DT & p-value \\
\hline $\mathrm{N}$ & 129 & 60 & \\
Adjusted OR Time & $0.94 \pm 0.2$ & $1.13 \pm 0.2$ & $<0.01$ \\
Age (years) & $49.1 \pm 15.0$ & $46.8 \pm 15.1$ & 0.33 \\
BMI* & $28.9 \pm 6.4$ & $29.0 \pm 6.4$ & 0.98 \\
Cancer & $28(21.7 \%)$ & $6(10.0 \%)$ & 0.07 \\
Complications & $17(13.2 \%)$ & $23(38.2 \%)$ & $<0.01$ \\
Compressive Symptoms & $51(39.5 \%)$ & $21(35.0 \%)$ & 0.55 \\
Female & $103(79.8 \%)$ & $44(73.3 \%)$ & 0.32 \\
Hashimoto's & $37(28.7 \%)$ & $6(10.0 \%)$ & 0.01 \\
History of Radiation & $3(2.3 \%)$ & $0(0.0 \%)$ & 0.55 \\
Hyperthyroid & $31(24.0 \%)$ & $38(63.3 \%)$ & $<0.01$ \\
MNG** & $23(17.8 \%)$ & $13(21.7 \%)$ & 0.53 \\
Number of Comorbidities & $1.9 \pm 1.5$ & $1.8 \pm 1.4$ & 0.57 \\
Pregnant & $1(0.8 \%)$ & $2(3.3 \%)$ & 0.24 \\
Previous RAI & $5(3.9 \%)$ & $5(8.3 \%)$ & 0.29 \\
Smoker & $12(9.3 \%)$ & $13(21.7 \%)$ & 0.02 \\
\hline
\end{tabular}

Data are expressed as a mean \pm standard error of the mean for continuous variables and the count with the percentage in parentheses for categorical variables.

$B M I=$ body mass index, $R A I=$ radioactive iodine, $O R=$ operative time. 


\section{Table 2}

\section{Subgroup Analysis}

\begin{tabular}{lccc}
\hline Variable & NDT & DT & p \\
\hline Hyperthyroidism & & & \\
N & 31 & 38 & \\
Age & $45.7( \pm 18.0)$ & $42.9( \pm 13.1)$ & 0.46 \\
Female & $27(87.1 \%)$ & $28(73.7 \%)$ & 0.17 \\
Ophthalmopathy & $3(9.68 \%)$ & $12(31.58)$ & 0.04 \\
Lugol's SSKI & $18(58.06 \%)$ & $31(81.58 \%)$ & 0.03 \\
a-TG antibody & $4(12.9 \%)$ & $13(34.21 \%)$ & 0.05 \\
Cancer & & & \\
N & 28 & 6 & \\
Age & $49.2( \pm 13.2)$ & $45.3( \pm 21.2)$ & 0.56 \\
Female & $18(64.3 \%)$ & $4(66.7 \%)$ & 0.89 \\
Thyroglobulin $(\mathrm{ng} / \mathrm{mL})$ & 55 & 897 & 0.03 \\
\hline
\end{tabular}

Data are expressed as percentage in parentheses for categorical variables.

$S S K I=$ saturated solution potassium iodide, $a-T G=$ anti-thyroglobulin antibody . 


\section{Table 3}

\section{Complications}

\begin{tabular}{lccc}
\hline Variable & NDT & DT & p \\
\hline N & 129 & 60 & \\
Complications & $\mathbf{1 7}(\mathbf{1 3 . 2 \%})$ & $\mathbf{2 3}(\mathbf{3 8 . 2 \%})$ & $<\mathbf{0 . 0 1}$ \\
Transient Hoarse & $7(5.4 \%)$ & $6(10.0 \%)$ & 0.23 \\
Permanent Hoarse & 0 & 0 & 1.00 \\
Transient & $\mathbf{1 0}(\mathbf{7 . 8 \%})$ & $\mathbf{1 4}(\mathbf{2 3 . 3 \%})$ & $<\mathbf{0 . 0 1}$ \\
Hypoparathyroid & & & \\
Permanent & 0 & 0 & 1.00 \\
Hypoparathyroid & & $\mathbf{5}(\mathbf{8 . 3 \%})$ & $<\mathbf{0 . 0 1}$ \\
Hematoma & $\mathbf{0}$ & &
\end{tabular}

Data are expressed as percentage in parentheses for categorical variables. Significant variables are in bold. 


\section{Table 4}

Multivariate Analysis

\begin{tabular}{lccc}
\hline Variable & Odds Ratio & $\mathbf{9 5 \%}$ CI & P \\
\hline Age & 1.00 & $0.97-1.02$ & 0.76 \\
Sex & 0.49 & $0.18-1.31$ & 0.16 \\
Hyperthyroid & $\mathbf{4 . 3 5}$ & $\mathbf{1 . 2 3 - 1 5 . 3 6}$ & $\mathbf{0 . 0 2}$ \\
a-TG antibody & $\mathbf{3 . 5 1}$ & $\mathbf{1 . 2 8}-\mathbf{9 . 6 6}$ & $\mathbf{0 . 0 2}$ \\
TG >150ng/mL & $\mathbf{2 . 6 1}$ & $\mathbf{1 . 0 6}-\mathbf{6 . 4 2}$ & $\mathbf{0 . 0 4}$ \\
Cancer & 0.72 & $0.22-2.33$ & 0.58 \\
Hashimoto's & 0.44 & $0.12-1.56$ & 0.20 \\
Smoker & 1.12 & $0.36-3.47$ & 0.85 \\
TSI & 0.70 & $0.20-2.49$ & 0.58 \\
\hline
\end{tabular}

Significant variables are in bold.

$a-T G=$ anti-thyroglobulin antibody, $T G=$ thyroglobulin, $T S I=$ thyroid stimulating immunoglobulin 